\title{
Spontaneous extrahepatic biliary tree perforation
}

\author{
T Pillay $^{1}$, V Ramkelawon ${ }^{2}$, B Singh ${ }^{1}$ \\ ${ }^{1}$ Department of General Surgery, University of KwaZulu Natal, Nelson R Mandela School of Medicine \\ ${ }^{2}$ Lenmed Ethekwini Hospital and Heart Centre, Durban
}

Corresponding author: TG Pillay (tharugp@yahoo.co.uk)

\begin{abstract}
Summary: Spontaneous perforation of the common bile duct is an extremely uncommon cause of an abdominal emergency. Owing to the rarity of the condition it is seldom suspected or diagnosed preoperatively. We report a case of a 42-year-old female presenting with features of severe biliary pancreatitis who responded well to organ support. Five days post admission she was deemed fit for cholecystectomy to prevent further attacks of pancreatitis. Surgery revealed a common hepatic duct perforation treated with cholecystectomy, lavage and common hepatic duct drainage. This was followed by postoperative biliary stenting which led to complete resolution without further surgery. This conservative staged approach obviated the need for major biliary reconstruction and is a viable strategy when confronted with this rare complication without prior ductal imaging.
\end{abstract}

Key words: Common bile duct perforation, spontaneous, biliary peritonitis

S Afr J Surg 2019;57(1)

http://dx.doi.org/10.17159/2078-5151/2018/v57n1a2770

\section{Case report}

A 42-year-old HIV negative female presented with a 1-day history of epigastric pain, nausea and vomiting. The medical history included hypertension, systemic lupus erythematosus and chronic renal dysfunction. In addition to anti-hypertensive therapy, the patient was being treated with plasmoquine and oral prednisone. On examination, she was ill looking and dehydrated. Abdominal examination revealed mild epigastric tenderness but no signs of peritonitis.

Abdominal radiographs showed no dilated bowel, air fluid levels or pneumoperitoneum and the psoas shadows were preserved. Abdominal sonography revealed gallstones and a dilated common bile duct (CBD), measuring $12 \mathrm{~mm}$; neither pericholecystic fluid, thickened gallbladder wall nor choledocholithiasis was reported. The pancreas and spleen were reported to appear normal. Only a sliver of fluid was noted in the pouch of Douglas. Initial laboratory investigations showed markedly elevated pancreatic enzymes (serum amylase $454 \mathrm{IU} / \mathrm{L}$ and serum lipase $2482 \mathrm{IU} / \mathrm{L}$ ), renal dysfunction (urea $13.4 \mathrm{mmol} / \mathrm{L}$, creatinine 297 umol/L), anaemia (haemoglobin $10.2 \mathrm{~g} / \mathrm{dL}$ ), mild thrombocytopenia (126 X10 $/ \mathrm{L})$, normal leucocyte count, raised liver enzymes (ALP 227 IU/L, GGT 140 IU/L, ALT 266 IU/L, AST 265 IU/L) with normal bilirubin levels (total bilirubin 11 umol/1 and conjugated bilirubin $7 \mathrm{umol} / \mathrm{l}$ ) and raised C-reactive protein (CRP) $48.4 \mathrm{mg} / \mathrm{L}$.

The clinical picture was consistent with gallstone pancreatitis and she was treated appropriately. The differential diagnoses included cholecystitis and cholangitis. Acute calculous cholecystitis was less likely in view of the negative ultrasound findings and absence of clinical findings to support the diagnosis. Cholangitis was considered unlikely given the absence of jaundice or marked elevation in transaminase levels. Within 12 hours of admission, the patient developed a severe systemic inflammatory response precluding magnetic resonance cholangiopancreatography (MRCP). The patient received aggressive fluid resuscitation and was commenced on inotropes and antibiotics. A septic screen (which included a chest radiograph, blood and urine cultures, and Fungitell assay) failed to identify any other source of sepsis. Her clinical condition improved over the next 4 days reflected by an improving trend of the liver enzymes and inflammatory markers. Serial abdominal examinations elicited only mild epigastric and right upper quadrant tenderness. Abdominal distension, palpable gallbladder and peritonism were not features. Early enteral nutrition was well tolerated.

Severe acute pancreatitis and acute cholecystitis remained likely diagnoses and as the patient was considered to have responded well to management, cholecystectomy and common bile duct exploration (CBDE) became a priority to prevent further episodes of pancreatitis. The patient was scheduled for a laparoscopic cholecystectomy and CBDE on day 5. At laparoscopy, $400 \mathrm{ml}$ of free bile was suctioned from the peritoneal cavity. The gallbladder, stomach and duodenum appeared intact. A leak from the common hepatic duct (CHD) was suspected and the procedure converted to laparotomy. A perforation of the proximal CHD measuring approximately $4 \mathrm{~mm}$ was noted and biopsied (Figure 1). 


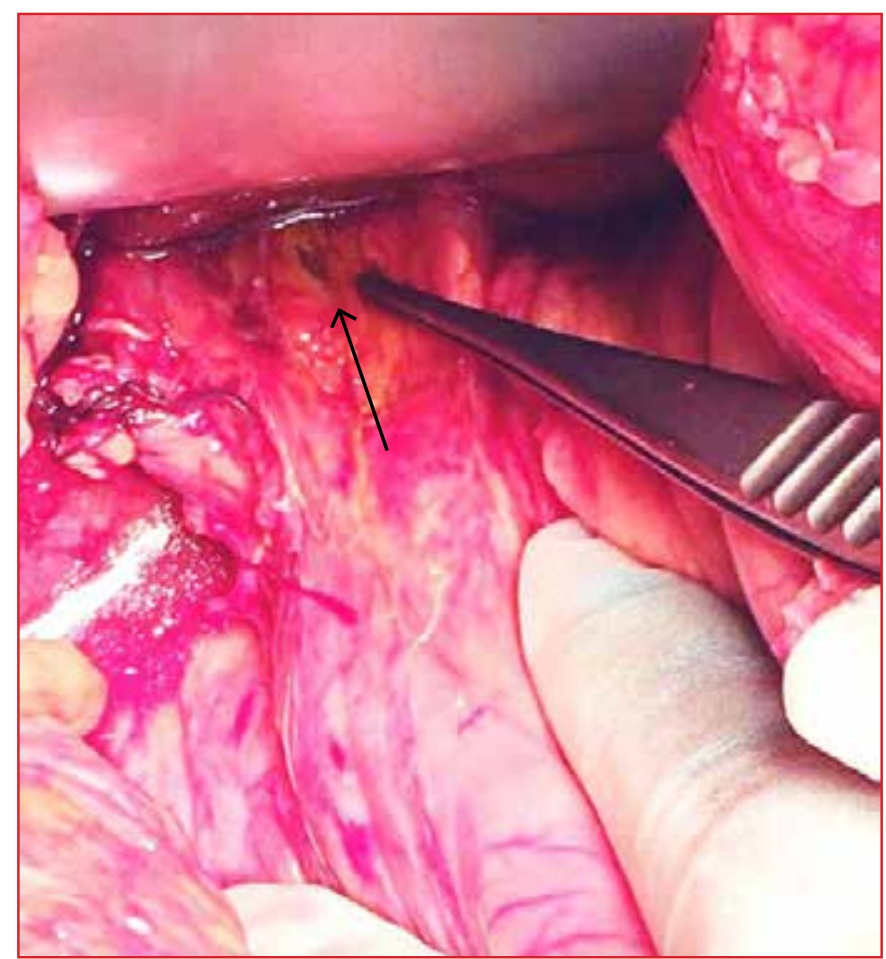

Figure 1. Intraoperative image demonstrating site of perforation (arrow)

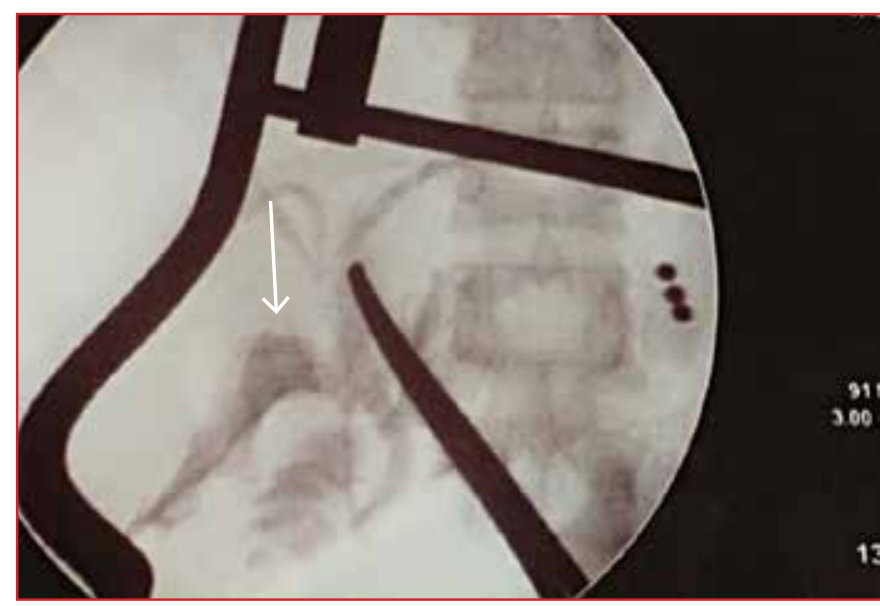

Figure 2. Intraoperative cholangiogram demonstrating absence of distal obstruction. Instrument tip points to site of perforation with contrast pooling is subhepatic space (arrow)

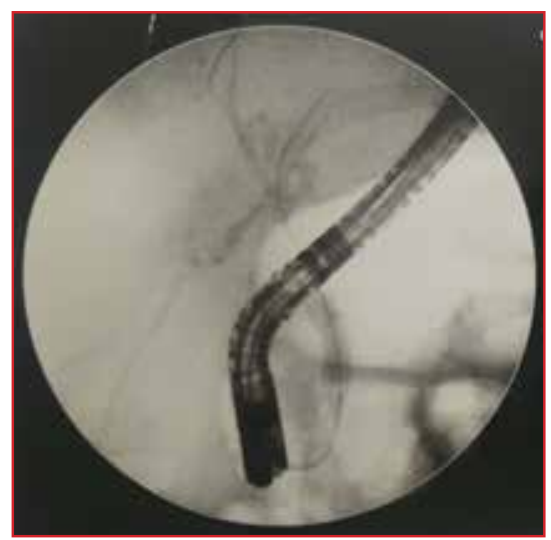

Figure 3.

Postoperative ERCP demonstrating contrast leak (pre stenting)
Intraoperative cholangiography (performed via the perforation using a size $8 \mathrm{FG}$ infant feeding tube) confirmed ductal anatomy and excluded distal obstruction (Figure 2). As there was no T-tube immediately available, no attempt at repair of the perforation was made. Peritoneal lavage was performed. The bile duct was drained externally using an 8 FG infant feeding tube and wide drainage of the subhepatic space was accomplished with use of a Yeates drain. Cholecystectomy was completed uneventfully.

Endoscopic retrograde cholangiopancreatography (ERCP) on postoperative day 5 (Figure 3), confirmed a leak at the level of the proximal common hepatic duct. A size 8.5 Fr plastic biliary stent was deployed across the perforation. Drainage of bile decreased progressively and the patient's condition continued to improve. The sub-hepatic drain was removed 7 days after biliary stenting. ERCP 6 weeks later confirmed absence of a leak, prompting removal of the stent.

The histology of the site of CHD perforation demonstrated necrotic fibroconnective tissue and excluded malignancy and granulomatous inflammation. Gallbladder histology revealed cholelithiasis with transmural inflammation and mucosal necrosis. Peritoneal fluid culture was negative and no malignant cells were found on cytology.

The patient made an uneventful recovery and was well at 6-month follow-up. She will continue to be followed up annually.

\section{Discussion}

Spontaneous biliary tree perforation is an exceptionally rare clinical entity. Since the first documentation of this entity in 1882 by Freeland, ${ }^{1}$ a further 90 adult cases have been reported. ${ }^{1,2}$ Perforation of the bile duct is far more common in the paediatric population due to congenital anomalies. ${ }^{3}$

Pathogenesis of the extrahepatic biliary tree perforation is not completely understood, although distal obstruction as well as wall weakness or a combination of these factors in conjunction with biliary calculous disease has been strongly implicated. ${ }^{4}$ Calculi are often associated with obstructive jaundice and biliary-enteric fistulae; these conditions also give rise to increased intraductal pressure and if a concomitant weakness of the ductal wall is present, the elevated pressure can lead to perforation. ${ }^{3}$ Intraductal infection, with or without intramural thrombosis, contributes to wall weakness and in combination with calculi, provides an environment for ductal perforation.

Other conditions implicated in spontaneous perforation include connective tissue disorders, rupture of a diverticulum, choledochal cysts, birth trauma, choledochal tuberculosis, HIV, parasites, pancreatic reflux, elevated intraductal pressure following sphincter of Oddi dysfunction, tumours, and biliopancreatic junctional pathology. ${ }^{1,3-7}$ Idiopathic perforations may be due to arterial thrombosis and poor ductal perfusion resulting in ischaemia, necrosis and subsequent perforation. ${ }^{8}$ We postulate that the aetiology in our patient was multifactorial as she had underlying connective tissue disease, 
was on chronic steroid therapy, likely had pancreatic reflux, intra-ductal infection and increased intra-ductal pressure due to a temporary gallstone impaction or oedema at the ampulla of Vater.

The clinical presentation of spontaneous perforation of the extrahepatic biliary tree may be acute or insidious, the latter being associated with abdominal distension, progressive jaundice, acholic stool and features of perihepatic abscess. The less common acute presentation is characterised by systemic inflammation, overt peritonitis and septicaemia. ${ }^{6}$ The case we report was of the acute type, however the clinical signs and inflammatory response were clouded by chronic immune-suppressive therapy, co-existing pancreatitis and cholecystitis. Given that there was no evidence of perforation on admission, we must assume that bile duct perforation occurred sometime thereafter. Peak levels of PCT and CRP were observed on day 3 .

Whilst ultrasonography and computed tomography scan will demonstrate intraperitoneal collections as well as identify cholelithiasis, these studies maybe non-specific and fail to provide a diagnosis. ${ }^{3}$ Hepatic scintigraphy has shown promise in terms of specificity and sensitivity for spontaneous ductal perforation. ${ }^{6}$

Extensive preoperative investigations will depend on patient stability and the need for urgent surgery. Laparoscopy has emerged as a useful tool in diagnosis and therapy with a diagnostic accuracy of $93-100 \%$ and achieving definitive treatment in up to $73 \%$ of cases. ${ }^{3}$

The recommended management approach will depend on the availability of pre- or intraoperative cholangiography. Should the cholangiogram reveal no distal obstruction, a primary repair of the perforation over a T-Tube is acceptable, provided the width of the perforation does not exceed $50 \%$ of the circumference of the bile duct. ${ }^{9}$ The use of a T-tube in a small calibre duct is not recommended and may be associated with increased incidence of stricture formation; therefore some experienced biliary surgeons may prefer to perform a biliary enteric bypass versus simple repair without a T-Tube when faced with this situation. ${ }^{10}$ Larger perforations are best managed with creation of a biliary enteric bypass. In the scenario of a distal obstruction, a biliary enteric bypass is performed. ${ }^{9}$ If cholangiography facilities are not readily available, the recommended surgical approach is T-tube or simple fine bore catheter drainage of the bile duct with cholecystectomy, as was performed in this case. Any attempt at more extensive surgery may significantly contribute to morbidity without benefit. ${ }^{1,4-6,11}$ In all cases wide drainage of the sub-hepatic space is mandatory. Biopsy of the perforation site is important to exclude a systemic cause for the perforation.
In the unusual event of diagnosing a spontaneous bile duct perforation preoperatively, the priority is to control any sepsis with US guided, laparoscopic, or open drainage and lavage as dictated by the patient's clinical condition and radiology findings. ERCP, sphincterotomy and bile duct stenting may be employed either preoperatively or postoperatively as an adjunct to radiological or surgical intervention.

\section{Conclusion}

Spontaneous biliary ductal perforation is an extremely rare condition with the potential for morbidity and mortality. Endoscopic bile duct stenting is highly attractive and feasible as an adjunct to a safe conservative surgical approach employing wide drainage, and may obviate the need for extensive surgery and complex bile duct reconstruction which is best left to an experienced biliary surgeon. Biopsy of the perforation site is advisable to exclude a systemic cause.

\section{REFERENCES}

1. Shawky MAE, Hassan AM, Mohammed MF, Moustafa HM, Mohammed AEAE, Eid MAE. Spontaneous common bile duct perforation in an adult female patient presenting with severe epigastric pain mimicking acute pancreatitis. Al Azhar Assuit Med J. 2016;(14:46-8.

2. Dash AP, Mishra T, Mohapatra M, Ibrahullah MD. Spontaneous perforation of bile duct. Trop Gastroenterol. 2012;33(2):151-3.

3. Sharma C, Desale J, Waghmare M, Shah H. A Case of Biliary Peritonitis following Spontaneous Common Bile Duct Perforation in a Child. Euroasian J Hepatogastroenterol. JulDec 2016; 6(2):167-9.

4. Khanna R, Agarwal N, Singh AK, Khanna S, Basu SP. Spontaneous Common Bile Duct Perforation Presenting as Acute Abdomen. Indian J Surg. 2010;72(5):407-8.

5. Subasinghe D, Udayakumara EA, Somathilaka U, Huruggamuwa M. Case Rep Gastrointest Med. 2016;2016:5321304. doi: $10.1155 / 2016 / 5321304$.

6. Mohanty SK, Mahapatra T, Behara BK, Acharya B, Kumar S, Dash JR, et al. Spontaneous perforation of common bile duct in a young female: An intra-operative surprise. Int J of Surg Case Reports. 2017;35:17-20.

7. Jarmin R, Alwi RI, Shaharuddin S, Salleh KM, Gunn A. Common Bile Duct Perforation Due to Tuberculosis: A Case Report. Asian J Surg. 2004;27(4):342-4.

8. Bediako-Bowan AAA, Dakubo JCB, Asempa M. Spontaneous extra-hepatic bile duct perforation postpartum. Ghana Med J. 2013;47(4):204-7.

9. Bhattacharjee PK. Approach to a Patient with Post Laparoscopic Cholecystectomy Bile Leak. Surgery. 2017;13(4):109-13. doi: 10.7438/1584-9341-13-4-1

10. Connor S, Garden OJ. Bile duct injury in the era of laparoscopic cholecystectomy. Br J Surg. 2006;93:156-8.

11. Kobayashi K, Kushida N, Ookubo S, Sano Y, Oomori H, Ohashi $\mathrm{H}$, et al. Bile Peritonitis due to spontaneous perforation of the Left hepatic Duct: A case report. JMAJ. 2005;48(8):422-5. 